\title{
ENCEPHALOID DISEASE OF THE HEART,
}

\author{
WITH OBSERVATIONS.
}

By PRESCOTT HEWETT,

LECTURER ON ANATOMY AT ST. GEORGE'S HOSPITAL MEDICAL SCHOOL.

Received April 20th-Read Nov. 10th, 1846.

As the following two cases present, I think, points of interest not unworthy of consideration, both in a pathological and in a practical view, $I$ trust that they will be acceptable to the Society.

Case 1.-Benjamin Rooth, ætat. 40, was admitted into St. George's Hospital, under the care of Mr. Keate, on the 15th of May 1844, with a large tumour, apparently of a carcinomatous nature, connected with the posterior part of the left foot. The disease had begun, about a year before the patient's admission into the hospital, by pain in the heel, for which he could assign no cause; this pain was followed by swelling of the part, which, within the last six months, had been rapidly increasing in size.

The patient, although somewhat reduced, stated that he enjoyed good bodily health; his countenance was somewhat pale, his pulse natural, his respiration free, and no œdema was observed about the extremities. At a consultation of the surgeons, it was determined that the leg should be removed, as there were no symptoms of visceral disease. Mr. Keate performed the operation on the 25th of the month, and upon examining the tumour it proved to be of an encephaloid nature, the bones and soft tissues of the back part of the foot 
being implicated in the disease, which had apparently begun in the os calcis.

For the first two days after the operation, the patient appeared to be doing very well; but on the third day there was slight irritative fever, and the stump had put on an unhealthy appearance: on the following day he was in a very low state, with an intermittent pulse, and complained of pain in the right hypochondrium. These symptoms went on increasing; jaundice, profuse perspirations and severe pleuritic pain of the left side, made their appearance on the 30th, and the patient died on the 31 st.

The body was examined thirteen hours after death. Marks of extensive recent inflammation, of a low character, were found in the left pleura, and in the pericardium. Slight emphysema existed at the anterior part of the lungs, and, at the posterior part, these organs were congested, but they did not present any other morbid appearance. The heart, much increased in size, presented dilatation of the right cavities, with hypertrophy of the muscular structure of the left ventricle. In the right auricle was a large growth, which, proceeding from the appendix, occupied the greater part of the cavity of the auricle, and, passing through the auriculo-ventricular opening, projected into the ventricle, reaching nearly to the bases of the columnæ carneæ of the valve.

The shape of this growth was conical, with its basis towards the auricle ; it distended the cavity of the appendix, in which it appeared to have originated, and it was also connected with that part of the auricle in the immediate neighbourhood of the appendix, but it was impossible to decide which of the tissues of this organ had been the primary seat of the disease. The surface of the tumour was irregular, and its tissue firm, except at the lower part, where it was somewhat flocculent: when cut into, it presented a highly vascular appearance, and in structure resembled exactly the encephaloid disease of the foot, for which the patient's leg had been removed. The valves of the heart were healthy in structure, and all the cavities of this organ contained coagula of blood, for the 
greater part fibrinous. No morbid appearance was observed about the viscera of the abdomen. Some enlarged glands were found in the groin, but they did not contain any carcinomatous deposit. The stump presented an unhealthy appearance, and one of the posterior tibial veins,-which, at its extremity, was open to the extent of half an inch, contained soms pus, above which were coagula, apparently of a healthy nature; the other vessels did not present any morbid appearance. No carcinomatous disease was discovered in any other part of the body.

The growth found in the right auricle was not examined by the microscope, but its general characters were so well marked that $I$ have no doubt as to its encephaloid nature.

Case 2.-Mary Hudson, ætat. 59, was admitted into St. George's Hospital, under the care of Mr. Keate, on the 10th of December 1845, with a tumour of the right breast, as large as the egg of an ostrich. The disease had existed about six months, and made its appearance after a blow on the breast, connected with a fall. The tumour, which was very painful, presented evident fluctuation in front, but at the back part it was solid; to its outer side was another tumour, of the size of a pullet's egg, firm in structure and apparently connected with one of the absorbent glands; the axillary glands were slightly enlarged; a red blush, of an erysipelatous character, existed in the skin covering the tumour, and had spread to some distance. The countenance was anæmic and of a yellowish colour; the respiration free, and the pulse weak, but otherwise natural. The patient, who was cheerful, presented no symptoms of disease beyond that of the breast.

On the 14th a grooved needle was passed into the front part of the tumour, and a pint of straw-coloured serum was let out; another puncture was then made at the back part of the tumour, but no fluid followed. On the 18th, the breast being as large as before, a fresh puncture was made in the neighbourhood of the first, but this time a small quantity only of blood and serum escaped. 
About three weeks after the admission of this patient into the hospital, a consultation of the surgeons was held, at which it was determined, under existing circumstances, not to meddle with the breast. At this time the patient was in good spirits, and there were no symptoms whatsoever to draw attention to any organic disease of any one of the internal viscera. By degrees the tumour gradually increased in size; the skin covering it assumed a duskier hue, and ultimately gave way at the points where the punctures had been made; the ulceration spread, and a large fungating growth made its appearance. She continued in this state, but gradually getting weaker, until the 20th of January 1846, when, at halfpast five in the afternoon, she was suddenly attacked with urgent dyspnœa and partial syncope, the pulse at the wrist being scarcely perceptible; these symptoms increased, notwithstanding the various remedies made use of, and she died on the following day at a quarter to one.

At the examination of the body, twenty-two hours after death, the tumour of the breast proved to be of a decidedly encephaloid nature; it was remarkably soft, almost fluctuating, and, in several places, presented the peculiar yellow opaque appearance so commonly observed in these tumours. Several of the absorbent glands were affected; one of them, of the size of a large egg and situated close to the original tumour, presented a well-marked specimen of this disease. A small encephaloid tumour was also found in the left rectus abdominis, the muscular structure of which it had partially destroyed. The cavities of both pleuræ contained a quantity of clear serum, which in the left amounted to about a pint; and, on the right side, there were some old adhesions. The lungs were quite healthy, with the exception of trifling emphysema at the anterior part, and slight congestion at the posterior part. The heart was enlarged by dilatation of its cavities, which on the right side were otherwise healthy. The dilatation of the left auricle was more marked than that of the other cavities; the mitral valve was slightly contracted, and there was some thickening of its tendinous chords : the 
auricular surface of this valve was, for the greater part, covered over by a soft deposit, pinkish in colour, of an irregular shape, resembling that of a cauliflower, and, to the naked eye, presenting exactly the appearance of the mass of the encephaloid disease observed about the breast and glands. This species of vegetation projected some distance, both into the auricle and into the ventricle, running along the surface of the tendinous chords, and covering the extremities of the muscular columns ; and by it the auriculo-ventricular opening was all but clogged up, there being merely an opening, of the size of a quill, left for the passage of the blood. Portions of the diseased structure were easily scraped off with a knife, but the mass of it was firmly connected with the parts upon which it was situated. The aortic valves were slightly thickened, and two of them had, at their apices, become united to each other. No encephaloid disease existed in any other part of the heart, and no morbid appearance was observed about the coagula contained in the cavities of this organ. The abdominal viscera were healthy. No odema existed about the extremities.

The portions of the diseased structure, both of the breast and of the heart, which were examined by the microscope, presented some large nucleated cells, of a spherical shape, with a quantity of granular matter floating about, but no caudate cells were observed in either of the specimens.

Both the preceding cases present rare forms of carcinomatous affections of the heart, but it is to the latter of them that I am more especially desirous of calling the attention of the Society, as in this case there existed a well-marked specimen of encephaloid disease of the free surface of the endocardium, an affection rarely met with,-so rarely indeed, that Dr. Walshe,* in his elaborate treatise on "Cancer," lately published, after stating his opinion that "there is nothing repulsive to reason in the idea of limitation of cancerous disease to the endocardium, but that if such cases really occur

* Nature and Treatment of Cancer, 1846, p. 371. 
they are singularly rare,"-alludes to one case only, which he quotes from the Revue Médicale, and even of the true nature of this case he expresses very great doubt; an opinion which is, I think, most fully warranted by the description of the morbid appearances.

The species of vegetation which was found on the mitral valve of Mary Hudson presented, to the naked eye, all the characters of encephaloid disease, and, when compared with portions of the diseased breast and gland, the resemblance was striking. The disease with which such a vegetation was most likely to be confounded is that form of soft fibrinous deposit so commonly observed about the surface of the endocardium, but the microscopical examination of this vegetation at once pointed out that such was not the nature of this deposit. Its general appearance, and the circumstances under which it occurred, led me to think that it was of an encephaloid nature, and the opinion which I then formed was subsequently strengthened by the following observation from Rokitansky:* "In rare cases, and under the requisite constitutional conditions, the vegetations on the valves of the heart may be of a cancerous, or more especially of a medullary, nature."

I avoid entering into a discussion concerning the origin of the encephaloid disease of the heart, in the latter case, as this would be foreign to my present purpose.

With regard to the first case, it is impossible to decide whether the disease began in the lining membrane of the appendix, or in its subjacent structures; but, whatever its origin, the fact of a tumour of this size, projecting from the right auricle into the ventricle and occupying so large a portion of the auriculo-ventricular opening, without ever producing symptoms sufficiently marked to call attention to the circulating organs, is certainly interesting, and only to be explained by the tumour growing slowly, and thus allowing the parts to accommodate themselves to its presence.

In a practical view, the two cases present, $I$ think, points of

* Vol. ii. p. 507. 
great interest. In the first case, the patient underwent a most severe operation, without there being the slightest suspicion of the disease of the heart, no symptom being present which could in any way lead one to suppose that this organ was so seriously affected : every thing went on as usual at such operations, and the only wonder is, that, under so severe a trial, the function of the central organ of circulation was not interfered with, and that the man did not die on the operating table. A case somewhat similar, in which the disease was, however, more extensive, is given by Cruveilhier,* but in this case the man died suddenly after a hearty meal. In the second case, the question naturally arises as to how long the carcinomatous affection of the heart had existed, and whether it existed at the time of the consultation of the surgeons. Such a deposit as that which was found on the mitral valve, in this case, might take place very rapidly, but as the patient did not present, until within a few hours of her death, any symptoms of disease of the heart, it is impossible to say when this disease began.

The preparations illustrative of the two cases which form the subject of this paper, are in the Pathological Museum of St. George's Hospital.

- Anatomie Pathologique, liv. xxix. 